\title{
LOCAL CRITICAL PERTURBATIONS OF UNIMODAL MAPS
}

\author{
ALEXANDER BLOKH AND MICHAŁ MISIUREWICZ
}

\begin{abstract}
We introduce a new complete metric in the space $\mathcal{V}_{2}$ of unimodal $C^{2}$ maps of the interval, with two maps close if they are close in the $C^{2}$-metric and differ only on a small interval containing their critical points. We identify all structurally stable maps in the sense of this metric. They are maps for which either (1) the trajectory of the critical point is attracted to a topologically attracting (at least from one side) periodic orbit, but never falls into this orbit, or (2) the critical point is mapped by some iterate to the interior of an interval consisting entirely of periodic points of the same (minimal) period. We verify the generalized Fatou conjecture for $\mathcal{V}_{2}$ and show that structurally stable maps form an open dense subset of $\mathcal{V}_{2}$.
\end{abstract}

\section{INTRODUCTION}

One of the central problems in dynamical systems theory is to investigate how the dynamics of a map changes if the map is slightly perturbed. The size of a perturbation is normally measured by the $C^{r}$-distance between maps. The dynamics is said to change in an essential way if the new map is not conjugate to the original one. If a map $f$ has a neighborhood $U$ such that all maps in $U$ are conjugate to $f$ then $f$ is said to be structurally stable. The definition of structural stability depends on the topology in the space of maps, but since the standard agreement is that maps are considered with $C^{r}$-topology, this dependence is omitted from the definition. According to the generalized Fatou conjecture, structurally stable maps should form an open dense subset in the entire space and should have some nice properties (like hyperbolicity). The original Fatou conjecture goes back to Poincaré, Fatou and Andronov and was stated in [3] by Fatou for rational maps. For interval maps, density of Axiom A maps has been proved by Jakobson [4] in $C^{1}$-topology, and more recently, in $C^{r}$-topology $(r=2,3, \ldots, \infty, \omega)$ by Kozlovski [5] for unimodal maps and by Kozlovski, Shen and van Strien [6] for multimodal maps.

Let us look at this situation from a different point of view. Suppose that maps $g$ and $h$ are close to a map $f$ so that the $C^{r}$-distances between $g$ and $f$ and between $h$ and $f$ are the same. Suppose however that the map $g$ differs from $f$ on the entire space while the map $h$ differs from $f$ only on a small open set. Then intuitively one should declare that $h$ is closer to $f$ than $g$. To reflect this intuitive feeling one needs to introduce another metric in the space of all $C^{r}$-maps.

Now, suppose that $f$ is not structurally stable. Then there are maps with different dynamics which are very close to $f$ in the $C^{r}$-metric, and one can ask if these maps

Date: July 16, 2008.

2000 Mathematics Subject Classification. Primary: 37E05, Secondary: 37C75.

Key words and phrases. Unimodal maps, Axiom A, Structural stability.

The first author was partially supported by NSF grant DMS 0456748 and the second author by NSF grant DMS 0456526. 
differ from $f$ on big sets (like $g$ ) or on small sets (like $h$ ). Theoretically it might happen that to change the dynamics of a structurally unstable $f$ one needs to introduce small changes $f$ everywhere, while the dynamics of $f$ persists under small changes on small sets. If this were the case, it would reflect a version of stability of the dynamics of $f$. In our view, making a distinction between these two situations is natural and important.

We would like to employ this point of view in the context of smooth unimodal maps. Despite apparent simplicity, these maps provide a variety of hard to study dynamical phenomena. The crucial role for the description of their dynamics is played by the behavior of the trajectory of the critical point, on which the dynamics depends almost completely. Therefore, if we want to achieve the maximal impact by slightly changing the map on a small set, it is natural to choose the set to be a small neighborhood of the critical point. In other words, one can expect that the critical point is the most sensitive to the changes of the map and makes the greatest impact upon the dynamics. This motivates us to study the question of which unimodal maps change their dynamics under small perturbations on small neighborhoods of their critical points, and which are stable with respect to such perturbations. An additional motivation is the fact that this was the main kind of perturbations used by Jakobson in [4] and that it works for Collet-Eckmann maps in higher smoothness (see [1]).

We suggest a certain formalism which reflects the above considerations and may be helpful if we compare our approach to the standard one.

For a closed interval $I$ and a $C^{r}$-function $\varphi: I \rightarrow \mathbb{R}$ we will denote by $\|\varphi\|_{r, I}$ the $C^{r}$-sup-norm of $\varphi$; the corresponding metric is denoted by $d_{r, I}$. We want some smoothness, so we will assume that $r \geq 1$ and consider the space $C^{r}(I, \mathbb{R})$ of realvalued $C^{r}$-functions on $I$, as well as a subspace $C^{r}(I, J)$ of $C^{r}(I, \mathbb{R})$, consisting of the functions with values in an interval $J$. Without loss of generality, by a unimodal $C^{r}$-map we mean a map $f \in C^{r}(K, K)$ of a closed interval $K=[a, b]$ into itself such that it has a unique critical point $c=c_{f}, f$ attains a local maximum at $c_{f}$, and $f(a)=f(b)=a$ (we will keep the notation $K, a, b, c$ throughout the paper). Given an interval $I$, we denote its length by $|I|$.

Normally this space of maps is endowed with the $C^{r}$-metric and the corresponding topology. To implement the ideas described earlier, we introduce a finer metric. Let $f$ be a unimodal $C^{r}$-map and $g$ be its perturbation. ${ }^{1}$ Then denote by $\operatorname{Di}(f, g)$ the open set of all points $x$ such that $f(x) \neq g(x)$. The idea is to measure both how big $\operatorname{Di}(f, g)$ is and how far it is from the critical points of $f$ and $g$. To do so, let $I(f, g)$ be the smallest closed interval containing $\operatorname{Di}(f, g), c_{f}$ and $c_{g}$. Set $\rho_{r}(f, g)=|I(f, g)|+\|f-g\|_{r, K}$. Then $\rho_{r}(f, g) \geq\|f-g\|_{r, K}$. In the next section (Proposition 2.1) we will show that $\rho_{r}$ is a complete metric.

Observe that the metric $\rho_{r}$ is strictly finer than the $C^{r}$-metric. For instance, if $K=[0,1]$ and $f \in C^{r}(K, K)$ is unimodal then unimodal self-mappings of $K$ defined as $f_{\varepsilon}=(1-\varepsilon) f$ converge to $f$ in $C^{r}$-sense, while $\rho_{r}\left(f_{\varepsilon}, f\right)>|K|=1$ for any $\varepsilon$. Finally, one can introduce topology analogous to the $C^{\infty}$-topology in the space of all

\footnotetext{
${ }^{1}$ We will use the word "perturbation" basically in 2 different meanings. The first one is the usual "process of perturbing." The second one is as here: we start with a map $f$, perturb it, and get a map $g$ which is a perturbation of $f$. However, when we speak of a "small perturbation," we mean that $g-f($ not $g)$ is small.
} 
unimodal $C^{\infty}$-maps by declaring that $C^{\infty}$-maps converge to a map if they converge to it in all $\rho_{r}$-metrics.

Since the metric $\rho_{r}$ reflects closeness of maps around their critical points and coincidence far away from the critical points, it serves our purpose and will be used from now on. To distinguish between ours and standard terminology we speak of (arbitrarily) c-small perturbations meaning (arbitrarily) small perturbations in $\rho_{r}$-metric, c-structurally (un)stable meaning structurally (un)stable in $\rho_{r}$-metric, c-close meaning close in $\rho_{r}$-metric, and so on (it will follow from the context what smoothness $r$ we consider).

Let us fix a closed interval $K=[a, b]$. Think of trivial ways in which a c-small perturbation of a unimodal map $f$ is not topologically conjugate to $f$. One such way would be to have $f\left(c_{f}\right)$ or $f^{2}\left(c_{f}\right)$ to be equal to an endpoint of $K$, a property that can be easily destroyed by such a perturbation. Unless $f(a)=a$, this reflects the choice of $K$, rather that the dynamics of $f$ and its perturbations. However, we excluded such phenomena by requiring that $f(a)=f(b)=a$.

Another trivial situation when a c-small perturbation produces a map which is not topologically conjugate to $f$ occurs when the critical point of $f$ is degenerate, that is, $f^{\prime \prime}\left(c_{f}\right)=0$. Then there are c-small perturbations $g$ with $c_{f}$ as a critical point, but with $g^{\prime \prime}\left(c_{f}\right)>0$. Thus, $g$ is not unimodal, so it cannot be conjugate to $f$. This basically means that unimodal maps with a degenerate critical point should be considered c-structurally unstable. However, the metric $\rho_{r}$ that we are using, is not defined outside the space of unimodal maps. Fortunately, there is another point of view, that we will adopt here. Namely, we consider only perturbations that are unimodal. Note that if the critical point is non-degenerate, its $C^{2}$-c-small perturbation is also unimodal. Hence, our decision will influence only classification of unimodal maps with the degenerate critical point.

We will denote the space of unimodal $C^{r}$-maps of $K$ into itself by $\mathcal{V}_{r}$ and its subspace consisting of maps with the critical point non-degenerate (that is, with the second derivative in the critical point non-zero) by $\mathcal{U}_{r}$. Those spaces are considered with the metric $\rho_{r}$. We proved already that the space $\mathcal{V}_{r}$ is complete. As we will prove in Lemma $6.1, \mathcal{U}_{r}$ is its open dense subset.

We are interested in describing all c-structurally stable maps in $\mathcal{V}_{r}$ and proving that c-structurally stable maps are dense in $\mathcal{V}_{r}$. To do so we need a couple of definitions. An interval $I$ is said to be periodic of period $k>1$ if $f^{k}(I) \subset I$ and the interiors of $f^{i}(I)$ are pairwise disjoint for $i=0,1,2, \ldots, k-1$. If $f \in \mathcal{V}_{r}$ is a map such that either (1) the trajectory of $c_{f}$ is attracted to a topologically attracting (at least from one side) periodic orbit, but never falls into this orbit, or $(2) c_{f}$ is mapped by some iterate of $f$ to the interior of an interval consisting entirely of periodic points of the same (minimal) period, then we call $f$ stabilized. Moreover, in the case (1) we call $f$ attractively stabilized, and in the case (2) neutrally stabilized. Observe that in both cases $c_{f}$ is mapped by some iterate of $f$ to the interior of a periodic interval $I$ of period $k$ with $\left.f^{k}\right|_{I}$ monotone. Moreover, if $x$ belongs to such interval then either $x$ is periodic or the trajectory of $x$ is attracted to a topologically attracting (at least from one side) periodic orbit.

Denote the set of all stabilized maps in $\mathcal{V}_{2}$ by $\mathcal{S} t_{2}$. Our main result is the following theorem, in which we verify the generalized Fatou conjecture for $\mathcal{V}_{2}$. 
Theorem 1.1. A map $f \in \mathcal{V}_{2}$ is c-structurally stable if and only if it is stabilized. Moreover, $\mathcal{S} t_{2}$ is an open dense subset of $\mathcal{V}_{2}$.

Let us compare this theorem with a theorem of Kozlovski in which he verifies the generalized Fatou conjecture for unimodal maps.

Theorem 1.2 (Kozlovski). The set of real analytic unimodal maps whose critical points are attracted by attracting periodic orbits is dense in the set of all unimodal $C^{r}$-maps in the $C^{r}$-topology for $r=1,2, \ldots, \infty, \omega$.

We use this theorem in an essential way when we consider infinitely renormalizable unimodal maps in Section 5. However, a major tool in [5] are analytic maps, which implies that in [5] perturbations inevitably differ from the original map on the entire interval except for finitely many points. Therefore the arguments from [5] are not directly applicable to $\mathcal{V}_{2}$. On the other hand, if the question of density of structurally stable maps is considered for smaller spaces of maps (like, for example, analytic unimodal maps, space of real polynomials, etc.) then even the question of density in the sense of $\rho_{r}$ is not always reasonable because then the space with $\rho_{r}$-metric may become discrete (as in the cases mentioned above).

We deal with the problem in a step-by-step fashion. Section 2 contains preliminaries. In Section 3 we show that stabilized maps from $\mathcal{V}_{2}$ are c-structurally stable. In Section 4 we consider finitely renormalizable unimodal maps in $\mathcal{U}_{2}$ and prove that if they are not stabilized then they are not c-structurally stable. The decay of geometry established in [2] and [11] is crucial for our arguments. Then in Section 5 we obtain similar results for infinitely renormalizable maps in $\mathcal{U}_{2}$ (and even in $\mathcal{U}_{\infty}$ ). Finally in Section 6 we deduce the results for $\mathcal{V}_{2}$ from the results for $\mathcal{U}_{2}$.

When the paper was ready for submission, we learned about Mike Todd's thesis [13] in which, among others, a problem of stability of unimodal maps was considered. In the case of non-recurrent critical points the results of [13] are similar to ours (as we point out before Lemma 4.1, they are known). In the case of a recurrent critical point it is shown in [13] that if a unimodal map $f$ with recurrent critical point $c$ is analytic or such that $\omega(c)$ is minimal, then for any point $x \in \omega(c)$ arbitrarily small $C^{k}$-perturbations of $f$ supported on an arbitrarily neighborhood of $x$ may change the combinatorial type of $f$. Todd uses complex analytic tools based upon [5, 7] and obtains results related to ours. However, [13] covers only limited class of maps while we solve the problem for all $C^{2}$-unimodal maps.

\section{Preliminaries}

We start by proving the proposition promised in the introduction.

Proposition 2.1. The function $\rho_{r}$ is a complete metric in $\mathcal{V}_{r}$.

Proof. Let us show first that $|I(f, g)|$ understood as the distance between $f$ and $g$ is a metric. It is clear that $\mid I(f, g)) \mid$ is symmetric and reflexive. To prove the triangle inequality observe that $I(f, g) \cup I(g, h)$ is a closed interval (it is the union of two closed intervals, each of which contains $c_{g}$ ) outside which we have $f=g=h$. Moreover, $c_{f}, c_{g}, c_{h} \in I(f, g) \cup I(g, h)$. Hence $I(f, h) \subset I(f, g) \cup I(g, h)$ and the triangle inequality follows. Thus, $\rho_{r}$ as a sum of two metrics, is a metric. 
Let us now show that it is complete. Suppose that we have a Cauchy sequence (in the sense of $\rho_{r}$ ) of unimodal maps $f_{1}, f_{2}, \ldots$ Then it follows that there exists $f \in C^{r}(K, K)$ such that $f_{i} \rightarrow f \in C^{r}(K, K)$ in the sense of the $C^{r}$-metric. Let $I_{m}=\overline{\bigcup_{j=m}^{\infty} I\left(f_{j}, f_{j+1}\right)}$. Since for any $j$ the intervals $I\left(f_{j}, f_{j+1}\right)$ and $I\left(f_{j+1}, f_{j+2}\right)$ intersect each other (both contain $c_{f_{j+1}}$ ), $I_{m}$ is a closed interval. Since we deal with a Cauchy sequence in the sense of $\rho_{r}$, for every $\varepsilon>0$ there exists $m$ such that $\left|I\left(f_{m}, f_{i}\right)\right| \leq \varepsilon$ for every $i \geq m$. Hence $I_{m} \subset\left[c_{f_{m}}-\varepsilon, c_{f_{m}}+\varepsilon\right]$ and $\left|I_{m}\right| \rightarrow 0$. Since $I_{m} \supset I_{m+1} \ldots$ we see that $\bigcap_{m=1}^{\infty} I_{m}=\{c\}$ for some point $c$. Then $c=\lim _{m \rightarrow \infty} c_{f_{m}}$ is a local maximum of $f$.

Now, for every $x \neq c$ there is a neighborhood $U$ of $x$ and an integer $m$ such that $f$ coincides with $f_{m}$ on $U$ and $c_{f_{m}} \notin U$. Thus, $c$ is the only critical point of $f$, so $f$ is unimodal (note that in general unimodal $C^{r}$-maps may converge in the $C^{r}$-sense to a non-unimodal map, e.g., to a map with an interval on which it is constant). Therefore $f \in \mathcal{V}_{r}$ and $\left|I\left(f_{m}, f\right)\right| \rightarrow 0$. This proves that $\rho_{r}\left(f_{m}, f\right) \rightarrow 0$.

For a unimodal map $f: K \rightarrow K$ set $c=c_{f}, c^{\prime}=c$ and for $x \neq c$ define $x^{\prime}$ as the unique point not equal to $x$ with $f\left(x^{\prime}\right)=f(x)$. Observe that the map $x \mapsto x^{\prime}$ is defined on the entire $K$ because we assume that $f(a)=f(b)$. Given a set $A \subset K$, set $A^{\prime}=\left\{x^{\prime}: x \in A\right\}$. In what follows any interval $I \subset K$ such that $I^{\prime}=I$ will be called symmetric. We will also use the notation $c_{1}=f(c)$.

In order to make a c-small perturbation of a unimodal map, we will be adding to this map a small "bump" concentrated close to the critical point. The following lemma describes this procedure.

Lemma 2.2. Let an integer $r \geq 2$, a map $f \in \mathcal{V}_{r}$ on an interval $K$, and a symmetric interval $I \subset K$ be given. Then for every $\varepsilon>0$ there is $\delta>0$ such that if $z \in K$ is a point with $0<|z-f(c)|<\delta$ then there exists $g \in \mathcal{U}_{r}$ such that $f$ and $g$ coincide outside $I$, have the same critical point $c, g(c)=z$, and $\rho_{r}(f, g)<\varepsilon$. Moreover, if $f$ is of class $C^{\infty}$, then $g$ can be chosen also of class $C^{\infty}$.

Proof. Fix functions $\psi_{+}, \psi_{-}: \mathbb{R} \rightarrow \mathbb{R}$ of class $C^{\infty}$, equal 0 outside the interval $[-1,1]$, with $\psi_{+}^{\prime}(0)=\psi_{-}^{\prime}(0)=0, \psi_{+}^{\prime \prime}(0), \psi_{-}^{\prime \prime}(0)<0$ and such that $\psi_{+}>0$ on $(-1,1)$, while $\psi_{-}<0$ on $(-1,1)$. We will look for $g$ of the form

$$
g(x)=f(x)+\alpha \cdot \psi_{ \pm}\left(\frac{x-c}{\beta}\right)
$$

for some $\alpha, \beta>0$, where we choose $\psi_{+}$when $z>f(c)$ and $\psi_{-}$when $z<f(c)$. Clearly, if $f$ is of class $C^{\infty}$, then $g$ is also of class $C^{\infty}$.

We fix $\beta$ so small that $[c-\beta, c+\beta] \subset I$ and $\beta<\min (1, \varepsilon / 4)$. We have

$$
\left|g^{(i)}(x)-f^{(i)}(x)\right|=\left|\frac{\alpha}{\beta^{i}} \cdot \psi_{ \pm}^{(i)}\left(\frac{x-c}{\beta}\right)\right|
$$

for $i=0,1, \ldots, r$, so

$$
\rho_{r}(f, g)<\varepsilon / 2+\frac{\alpha}{\beta^{r}}\left\|\psi_{ \pm}\right\|_{r,[-1,1]} .
$$

If $\alpha$ is small enough then the right-hand side of (2.2) is smaller than $\varepsilon$. On the other hand, if $\delta>0$ is sufficiently small, we can choose the corresponding small $\alpha$ to satisfy $g(c)=z$ and, by $(2.2)$, to guarantee that $\rho_{r}(f, g)<\varepsilon$. Thus, it remains to prove 
that for a sufficiently small $\alpha$ the map $g$ is unimodal with the critical non-degenerate point $c$.

Since $\psi_{ \pm}^{\prime}(0)=0, \psi_{ \pm}^{\prime \prime}(0)<0$ and $f^{\prime \prime}(c) \leq 0$, we get $g^{\prime}(c)=0$ and $g^{\prime \prime}(x)<0$ for every $x$ in a small neighborhood $U=(y, z)$ of $c$ with $f^{\prime}(y)>0$ and $f^{\prime}(z)<0$. Since $\left|g^{\prime}(x)-f^{\prime}(x)\right|$ is very small, the function $g(x)$ has positive derivative if $x<c$ and negative derivative if $x>c$. Outside $U$, if $\alpha$ is sufficiently small, the sign of $g^{\prime}$ is the same as the sign of $f^{\prime}$. Thus, $c$ is the maximum of $g$ and $g$ is unimodal. This completes the proof.

Any perturbation from the above lemma (or the next one) will be called a bump perturbation.

The next lemma uses the construction from Lemma 2.2 but includes some additional estimates and is proven only in $C^{2}$.

Lemma 2.3. Let $f \in \mathcal{U}_{2}$ and suppose that $\gamma>0$ is given. Then there exists $\delta>0$ such that for any interval $T=\left[a, a^{\prime}\right]$ with $|T| \leq \delta$ and any point $d \in K$ with $\frac{\left|d-c_{1}\right|}{|f(T)|} \leq \delta$ there exists a unimodal map $g \in \mathcal{U}_{2}$ with the same critical point $c$ such that $g=f$ outside $T, \rho_{2}(f, g) \leq \gamma$ and $g(c)=d$.

Proof. We use the same construction as in Lemma 2.2, but only with $\psi=\psi_{+}$and admitting $\alpha<0$. Thus, we look for $g$ of the form

$$
g(x)=f(x)+\alpha \cdot \psi\left(\frac{x-c}{\beta}\right)
$$

for some $\alpha, \beta \in \mathbb{R}, \beta>0$. According to (2.1) and (2.2), we have

$$
\left|g^{\prime \prime}(x)-f^{\prime \prime}(x)\right| \leq \frac{|\alpha|}{\beta^{2}} \zeta
$$

for all $x \in K$, and

$$
\rho_{2}(f, g) \leq 2 \beta+\frac{|\alpha|}{\beta^{2}} \zeta
$$

where $\zeta=\|\psi\|_{2,[-1,1]}$.

Since $f^{\prime \prime}(c) \neq 0$, there exists $\sigma>0$ such that for any interval $T=\left[a, a^{\prime}\right]$,

$$
|f(T)| \leq \sigma\left(\min \left(c-a, a^{\prime}-c\right)\right)^{2},
$$

and there exists $\tau>0$ such that $\left|f^{\prime \prime}(x)\right|>\tau$ for any $x \in(c-\tau, c+\tau)$. Set

$$
\delta=\min \left(\frac{\gamma}{4}, \frac{\gamma}{2 \zeta \sigma}, 1, \tau, \frac{\tau}{\sigma \zeta}\right)
$$

and if $T=\left[a, a^{\prime}\right]$ is an interval with $|T| \leq \delta$ which we fix from now on, set

$$
\beta=\min \left(c-a, a^{\prime}-c\right) \text {. }
$$

Note that

$$
\beta \leq|T| \leq \delta \leq \min (1, \tau) \text { and }|f(T)| \leq \sigma \beta^{2} .
$$

Take a point $d \in K$ with $\frac{\left|d-c_{1}\right|}{|f(T)|} \leq \delta$, and set $\alpha=d-c_{1}$. Then $g(c)=f(c)+\alpha=d$ and (in view of (2.5) and (2.6))

$$
\frac{|\alpha|}{\beta^{2}} \zeta \leq \frac{\left|d-c_{1}\right| \zeta \sigma}{|f(T)|} \leq \delta \zeta \sigma \leq \min \left(\frac{\gamma}{2}, \tau\right)
$$


To see that $g$ is unimodal, observe that $g=f$ outside $(c-\delta, c+\delta) \subset(c-\tau, c+\tau)$, and for $x \in(c-\tau, c+\tau)$ we have

$$
\left|g^{\prime \prime}(x)\right| \geq\left|f^{\prime \prime}(x)\right|-\left|f^{\prime \prime}(x)-g^{\prime \prime}(x)\right|>\tau-\left|f^{\prime \prime}(x)-g^{\prime \prime}(x)\right|,
$$

and this is positive by (2.3) and (2.7). Moreover, both $f$ and $g-f$ have a critical point at $c$, so $c$ is also a critical point of $g$.

Finally, by $(2.4)-(2.7)$

$$
\rho_{2}(f, g) \leq 2 \beta+\frac{|\alpha|}{\beta^{2}} \zeta \leq 2 \delta+\frac{\gamma}{2} \leq \gamma
$$

This completes the proof.

If $f$ is a smooth piecewise monotone map then an interval $I$ is said to be a homterval if for every $n$ there is no critical point of $f^{n}$ in its interior. Clearly, a point belongs to the interior of a homterval if and only if it is not approximated by precritical points. We need the following theorem, proved in [10]. We state it here in a form convenient for us.

Theorem 2.4. Let $f \in \mathcal{U}_{2}$. If $J$ is a homterval then $J$ is eventually mapped into a periodic homterval. In particular, if $c$ is not periodic then the critical value $c_{1}$ is approximated by precritical points if and only if none of the following happens:

(1) $c_{1}$ is mapped to the interior of a periodic homterval by some iterate of $f$,

(2) $f(c)=b$ and $a$ is an endpoint of an invariant homterval.

If there exists a periodic interval $I$ containing $c$ of period greater than 1 then $f$ is said to be renormalizable. If there exists a number $k$ such that a periodic interval containing $c$ cannot be of period greater than $k$ then $f$ is said to be finitely renormalizable, otherwise $f$ is said to be infinitely renormalizable. An interval $T=[p, q] \subset K$ such that $c \in(p, q)$ and all forward images of $p, q$ miss $(p, q)$ is said to be nice (see $[8])$. We always assume that nice intervals are small and map off themselves. Fix a nice interval $T$.

Consider a point $x \in K$. Then we can define the first entry map $R_{T}$ into int $T$ as the first positive iterate of $f$ (if any) which maps $x$ into int $T$. Clearly, there are points at which $R_{T}$ is not defined. The set of all points on which $R_{T}$ is defined is the union $D_{T}$ of pairwise disjoint open intervals called domains of $R_{T}$. There is one exceptional domain of $R_{T}$, called central return domain of $T$ and defined as follows. Suppose that $c$ is recurrent. Then there exists the minimal positive integer $m$ with $f^{m}(c) \in \operatorname{int} T$. We can choose an open interval $J \ni c_{1}$ such that $f^{m-1}(\bar{J})=T$ and $\left.f^{m-1}\right|_{J}$ has no critical points. The interval $f^{-1}(J) \cap T$ is then called the central return domain of $T$ and is denoted by $\widehat{T}$; moreover, $f$ maps $\widehat{T}$ a 2 -to- 1 fashion onto $J$. Clearly, even though $T$ is not necessarily symmetric, its central return domain always is. Observe that the endpoints of $\widehat{T}$ are mapped by $f^{m}$ into one endpoint of $T$. Otherwise a domain $I$ of $R_{T}$ is mapped by $R_{T}$ diffeomorphically onto $T$.

The properties of domains of $R_{T}$ in the case when $T$ is nice are well known. For the sake of completeness we state them in the following lemma. It is partially proved in [9], page 341, partially is obvious.

Lemma 2.5. Let $T \ni c$ be a nice interval, and let $Q$ be a domain of $R_{T}$. Then for some $m$ the map $\left.R_{T}\right|_{Q}$ coincides with $\left.f^{m}\right|_{Q}$, the endpoints of $Q$ are mapped by $R_{T}$ 
to the endpoint(s) of $T$ and have the positive $f$-orbits disjoint from int $T$, and there are two possibilities: (1) $\left.R_{T}\right|_{Q}$ is monotone and $R_{T}(\bar{Q})=T$ (if $Q$ is not a central domain of $T$ ), or (2) $\left.R_{T}\right|_{Q}$ has only one critical point (if $Q$ is a central domain of $T)$. Moreover, intervals $Q, f(Q), \ldots, f^{m}(Q)=R_{T}(Q)$ are pairwise disjoint, and also disjoint from $T$, except for the case when $Q \subset T$, in which case the only nonempty intersection may be between $Q$ and $f^{m}(Q)=R_{T}(Q)$.

Finally we state a result which we rely upon considering the finitely renormalizable recurrent case in Section 4. It has been proven in [2] and [11], and establishes the decay of geometry for maps in $\mathcal{U}_{3}$. Moreover, the same result is true in $\mathcal{U}_{2}$, see [11] and [12]. To state it we need some definitions. Given intervals $I, M$ with $I \subset \operatorname{int} M$ we set

$$
\varepsilon(M, I)=\max \left\{\frac{|I|}{\left|M^{-}\right|}, \frac{|I|}{\left|M^{+}\right|}\right\},
$$

where $M^{-}$and $M^{+}$are components of $M \backslash I$, and if $\varepsilon(M, I)<\varepsilon$, we say that $I$ is $\varepsilon$-inside $M$.

Theorem 2.6. Let $f \in \mathcal{U}_{2}$ be a finitely renormalizable map with a recurrent and non-periodic critical point $c$. Then for any $\varepsilon>0$ there exists a nice interval $I \ni c$ such that the central return domain $\widehat{I}$ is $\varepsilon$-inside $I$.

\section{StABilized MAPs AND THEIR STRUCTURAL STABILITY}

Here we prove the simple direction of Theorem 1.1 and show that stabilized maps are c-structurally stable. We also state a useful (although trivial) lemma dealing with maps whose critical points are periodic.

Theorem 3.1. For $r=0,1, \ldots \infty$, if $f \in \mathcal{S} t_{r}$ then it is c-structurally stable in $\mathcal{V}_{r}$.

Proof. If $f \in \mathcal{S} t_{r}$ then two cases are possible. First suppose that $f$ is neutrally stabilized. Then for some minimal $m$ the point $f^{m}\left(c_{f}\right)$ belongs to an open interval $I$ consisting of periodic points of minimal period $k$. Choose pullbacks of $I$ along $c_{f}, \ldots, f^{m}\left(c_{f}\right)$ and denote them $I_{-1}, \ldots, I_{-m}$ with $c_{f} \in I_{-m}$. Choose a small neighborhood $U \subset I_{-m}$ of $c_{f}$. If a perturbation is sufficiently c-small then it will not change the map outside $U$, and on $U$ the new map $g$ will act so close to $f$ that $g(U) \subset I_{-m+1}, \ldots, g^{m}(U) \subset I$. Clearly, a homeomorphism which acts as the identity outside $\bigcup_{i=1}^{m} I_{-i}=H$ and inside $H$ simply maps intervals $g^{i}(U)$ onto the intervals $f^{i}(U)$ appropriately, conjugates $g$ and $f$ which shows that neutrally stabilized maps are c-structurally stable. A very similar argument shows that if a map is attractively stabilized then it is c-structurally stable too. We leave this case to the reader.

The next lemma deals with the case when the orbit of the critical point is periodic.

Lemma 3.2. Suppose that $f \in \mathcal{V}_{r}(r=1,2, \ldots, \infty)$ and the orbit of $c_{f}$ is periodic. Then $f$ is not c-structurally stable in $\mathcal{V}_{r}$, but arbitrarily c-close to $f$ there are attractively stabilized maps.

Proof. Let $n$ be the period of $c=c_{f}$. For any sufficiently c-small bump perturbation $g$ of $f$ the point $c$ is a critical point of $g$ and $g^{n}(c) \neq c$, so $g$ is not topologically conjugate to $f$. Moreover, if the perturbation is sufficiently c-small, the attracting 
periodic orbit of period $n$ persists and attracts the $g$-trajectory of $c$. Thus, $g$ is attractively stabilized.

\section{Finitely RENORMALizABle maps}

In this section we consider finitely renormalizable unimodal maps. The main result here is Theorem 4.3 in which we show that a finitely renormalizable $f \notin \mathcal{S} t_{2}$ is not structurally stable. We begin with the non-recurrent case (this result is known, but we include it for completeness).

Lemma 4.1. Let $f \in \mathcal{U}_{r}(r=2,3, \ldots, \infty)$ be a map with the critical point $c$ which is non-recurrent and none of the following happens:

(1) $c_{1}$ is mapped to the interior of a periodic homterval by some iterate of $f$,

(2) $f(c)=b$ and $a$ is an endpoint of an invariant homterval.

Then arbitrarily c-close to $f$ in $\mathcal{U}_{r}$ there are maps with critical periodic points.

Proof. By Theorem 2.4 there are precritical points arbitrarily close to $c_{1}$. Let us show that then there exists a neighborhood $U$ of $c$ such that arbitrarily close to $c_{1}$ there are precritical points whose trajectories never enter $U$ before they hit $c$.

Consider two cases. First, it may happen that there is a small neighborhood of $c$ which contains no precritical points. Then choose this neighborhood as $U$. Now, suppose that there are precritical points in any neighborhood of $c$. Since $c$ is nonrecurrent, we can choose a precritical point $y<c$ so close to $c$ that the orbit of $c_{1}$ is disjoint from $\left[y, y^{\prime}\right]$. Moreover, we can choose $y$ so that no point inside $\left(y, y^{\prime}\right)=U$ is mapped into $c$ before $y$. That is, the order (the time necessary to get to $c$ ) of every precritical point from $\left(y, y^{\prime}\right)$ is strictly higher than that of $y$ (and of $y^{\prime}$ ).

Let $V$ be a small neighborhood of $c_{1}$ and let $z$ be the precritical point of the smallest order $m$ in $V$. Let us show that the trajectory of $z$ never enters $U$ before it hits $c$. Suppose that for some $k<m$ we have $f^{k}(z) \in U$. By the choice of $U$ then $f^{k}\left(c_{1}\right) \notin U$ and hence there exists a point $t \in V$ such that $f^{k}(t)=y$ or $f^{k}(t)=y^{\prime}$. By the choice of $y$ it follows that $t$ is precritical and of smaller order than $z$, a contradiction. Hence the trajectory of $z$ misses $U$ before it hits $c$.

Now, for every bump perturbation $g$ of $f$ such that $\operatorname{Di}(f, g) \subset U$, the point $z$ found above is also precritical for $g$. By Lemma 2.2 we can choose $g$ such that it is of the same class as $f$, differs from $f$ on an arbitrarily small neighborhood $W \subset U$, and once $W$ is fixed, $g(c)=z$ (so $c$ is periodic for $g$ ) and $z$ is so close to $c_{1}$ that $g-f$ has as small $C^{r}$-norm as we want.

We get the following corollary to the above lemma.

Corollary 4.2. If $f \in \mathcal{U}_{r} \backslash \mathcal{S} t_{r}(r=2,3, \ldots, \infty)$ and $c$ is non-recurrent then $f$ is not c-structurally stable in $\mathcal{V}_{r}$.

Proof. We may assume that $f(c) \neq b$ as otherwise $f$ is obviously not c-structurally stable. If the orbit of $c$ is infinite then $c$ is not mapped into the interior of a homterval because $f \notin \mathcal{S} t_{r}$, and in view of Theorem 2.4 every infinite trajectory of a point in an interior of a homterval is attracted to a periodic orbit. Thus, in this case $f$ is not c-structurally stable by Lemma 4.1. If the orbit of $c$ is finite, then $c$ is preperiodic. If $k$ is its eventual period then again because $f \notin \mathcal{S} t_{r}$, there exists an arbitrarily c-small 
bump perturbation $g$ of $f$ for which $c$ is not preperiodic with the eventual period $k$ (this explains the definition of a neutrally stabilized map).

Now we deal with recurrent finitely renormalizable case.

Theorem 4.3. If $f \in \mathcal{U}_{2} \backslash \mathcal{S} t_{2}$ is finitely renormalizable then $f$ is not c-structurally stable. Moreover, arbitrarily c-close to $f$ there are maps $g$ with periodic critical points.

Proof. By Lemma 3.2 we may assume that $c$ is not periodic. By Lemma 4.1 we may assume that $c$ is recurrent. Suppose that $\gamma>0$ is given. Now, choose $\delta$ applying Lemma 2.3 to $f$ and $\gamma$. By Theorem 2.6 there exists an arbitrarily small nice interval $I=\left[x, x^{\prime}\right]$ such that its central return domain $\widehat{I}=\left[y, y^{\prime}\right]$ is $\delta$-inside $I$. It follows that if $I$ is sufficiently small then $\frac{|f(\widehat{I})|}{|f(I)|}<\delta$.

Now, by Lemma 2.3 there exists a map $g$ with $\rho_{2}(g, f)<\gamma$ and $g(c)=f(y)$. Since by Lemma 2.5 the $f$-orbit of $f(y)$ avoids $\left(x, x^{\prime}\right)$, we see that the $g$-orbit of $g(c)=f(y)$ avoids $\left(x, x^{\prime}\right)$ because $g=f$ outside $\left(x, x^{\prime}\right)$. Since $c$ is recurrent for $f$, it implies that $f$ and $g$ are not topologically conjugate. Consider a family of maps $g_{t}, 0 \leq t \leq 1$, defined as $g_{t}(x)=t f+(1-t) g$. Clearly, all these maps are unimodal $C^{2}$-maps with the same critical point $c$ coinciding with $g=f$ outside $\left(x, x^{\prime}\right)$. Since $f$ and $g$ are not topologically conjugate it follows that for some $t$ the point $c$ will be $g_{t}$-periodic. By the choice of $g$ we have $\rho_{2}\left(f, g_{t}\right)<\gamma$ which completes the proof.

\section{INFINITELY RENORMALIZABLE MAPS}

The main result of this section is based upon a simple observation that for infinitely renormalizable maps the restriction of the appropriate iterate of the map onto a small periodic interval can be viewed as a globally defined map. We will need the following simple and well known lemma.

Lemma 5.1. For closed intervals $I$ and $J$, a positive integer $r$ and a $C^{r}$-function $\psi: J \rightarrow \mathbb{R}$, the map $f \mapsto \psi \circ f$ from $C^{r}(I, J)$ to $C^{r}(I, \mathbb{R})$ is continuous.

We will also need the following lemma about a specific extension of a function defined on an interval onto a greater interval.

Lemma 5.2. Let $r$ be a positive integer and let $I, J, T$ be closed intervals such that $I \subset \operatorname{int}(J) \subset J \subset \operatorname{int}(T)$. Then there exists a constant $M=M_{r}(I, J)$ such that the following holds. Given two functions $f \in C^{r}(T, \mathbb{R}), h \in C^{r}(I, \mathbb{R})$ there exists a function $F \in C^{r}(T, \mathbb{R})$ such that $\left.F\right|_{I}=\left.h\right|_{I},\left.F\right|_{T \backslash J}=\left.f\right|_{T \backslash J}$ and $\|F-f\|_{r, T}<$ $M\|h-f\|_{r, I}$. If $f$ and $h$ are of class $C^{\infty}$ then $F$ can be chosen of class $C^{\infty}$ except perhaps at the endpoints of $I$.

Proof. Consider the left endpoint $p$ of $I$ and the left component $L$ of $J \backslash \operatorname{int}(I)$. If $t=\|h-f\|_{r, I}$ then $\left|(h-f)^{(k)}(p)\right| \leq t$ for $k=0,1,2, \ldots, r$. For $x \in L$ set

$$
h(x)=f(x)+\sum_{k=0}^{r} a_{k}(x-p)^{k}, \quad \text { where } \quad a_{k}=\frac{(h-f)^{(k)}(p)}{k !} .
$$

This formula extends $h$ to a function of class $C^{r}$ on $I \cup L$ and

$$
\|h-f\|_{r, I \cup L} \leq(r+1) \max \left(1,|L|^{r}\right) t .
$$


We do the same construction with the right endpoint of $I$ and the right component of $J \backslash \operatorname{int}(I)$. In this way we get an extension of $h$ to a $C^{r}$-function on $J$ with

$$
\|h-f\|_{r, J} \leq(r+1) \max \left(1,|J|^{r}\right)\|h-f\|_{r, I} .
$$

Now we fix a function $\varphi: \mathbb{R} \rightarrow[0,1]$ of class $C^{\infty}$, depending only on $I$ and $J$, such that $\varphi$ is 1 on $I$ and 0 on a neighborhood of the closure of $\mathbb{R} \backslash J$. Finally, we define $F=f+\varphi(h-f)$. Clearly, $\left.F\right|_{I}=\left.h\right|_{I}$ and $\left.F\right|_{T \backslash J}=\left.f\right|_{T \backslash J}$. By the formulas for the derivatives of a product of two functions and by the estimate for $\|h-f\|_{r, J}$, there is a constant $M$, depending only on $r, I, J$, such that $\|F-f\|_{r, T}<M\|h-f\|_{r, I}$.

If $f$ and $h$ are of class $C^{\infty}$ then $F$ constructed as above is of class $C^{\infty}$ except perhaps at the endpoints of $I$.

Now we can prove the main result of this section. A closed interval $I$ will be called strongly periodic (of period $n$ ) if $I, f(I), \ldots, f^{n-1}(I)$ are pairwise disjoint while $f^{n}(I) \subset I$.

Theorem 5.3. Assume that $f \in \mathcal{U}_{r}(r \in\{2, \ldots, \infty\})$ is infinitely renormalizable. Then $f$ is not c-structurally stable. Moreover, arbitrarily c-close to $f$ there is a map $F$ for which the trajectory of its critical point is attracted to an attracting periodic orbit.

Proof. Fix $\varepsilon>0$. Since $f$ is infinitely renormalizable and by Theorem 2.4, there exists a positive integer $n$ and a closed strongly periodic interval $I \subset K$ of length less than $\varepsilon / 2$ and period $n$ such that $c=c_{f} \in \operatorname{int} I$. Then there exists a closed interval $J$ of length less than $\varepsilon$, containing $I$ in its interior and disjoint from $f^{k}(I)$, $k=1,2, \ldots, n-1$.

Assume first that $r$ is finite. By Theorem 1.2 there exists a $C^{\infty}$-map $g: I \rightarrow I$ arbitrarily $C^{r}$-close to $\left.f^{n}\right|_{I}$ and such that its critical point is attracted to an attracting periodic orbit. Since $\left.f^{n-1}\right|_{f(I)}$ is a diffeomorphism and $\left.f\right|_{I}=\left.\left(\left.f^{n-1}\right|_{f(I)}\right)^{-1} \circ f^{n}\right|_{I}$, the map $h: I \rightarrow f(I)$ defined as $h=\left(\left.f^{n-1}\right|_{f(I)}\right)^{-1} \circ g$ is arbitrarily $C^{r}$-close to $\left.f\right|_{I}$ by Lemma 5.1. By Lemma 5.2 we can extend $h$ to a $C^{r}$-map $F$ on $\mathbb{R}$ so that $F-f$ is supported on $J$ and is arbitrarily $C^{r}$-close to 0 . In particular, the sign of $F^{\prime}$ will be the same as the sign of $f^{\prime}$ outside of $I$. Since additionally we know that $\left.F\right|_{I}=\left.h\right|_{I}$ is unimodal, $F$ is also unimodal. Since $F=f$ on $f^{k}(I), k=1,2, \ldots, n-1$, we get

$$
\left.F\right|_{I}=\left.\left.f^{n-1}\right|_{f(I)} \circ h\right|_{I}=g,
$$

and therefore the critical point of $F$ is attracted to an attracting cycle.

If $r=\infty$ we first fix a finite $s$ and apply the above construction for $s$ instead of $r$ to get a map $F_{s}$ with $\rho_{s}\left(F_{s}, f\right)<1 / s$. By Lemma 5.2, we may assume that $F_{s}$ is of class $C^{\infty}$ except perhaps at the endpoints of $I$. Then a standard construction (like in the second part of the proof of Lemma 5.2) produces a map $G_{s}$ of class $C^{\infty}$, arbitrarily close to $F_{s}$ in the $C^{s}$-topology and coinciding with $F_{s}$ outside $J$ (which is shorter than $1 / s)$. By the definition of the $C^{\infty}$-topology the sequence $\left(G_{s}\right)_{s=1}^{\infty}$ converges to $f$ in this topology, which completes the proof for $r=\infty$.

\section{Proof of Theorem 1.1}

First we show how to deal with the maps with degenerate critical points.

Lemma 6.1. For every $r \geq 2$ the set $\mathcal{U}_{r}$ is open and dense in $\mathcal{V}_{r}$. 
Proof. Openness is clear, and density follows immediately from Lemma 2.2.

Now we can prove Theorem 1.1. One direction is immediate: if $f \in \mathcal{S} t_{2}$ then $f$ is c-structurally stable by Theorem 3.1.

Assume now that $f \in \mathcal{U}_{2}$ is c-structurally stable. If $f$ is finitely renormalizable, then it belongs to $\mathcal{S} t_{2}$ by Theorem 4.3. If $f$ is infinitely renormalizable, then by its c-structural stability and Theorem 5.3, it is conjugate to a map with the critical point attracted to an attracting periodic orbit. Thus, the same property has to hold for $f$. This prevents infinite renormalizability, so this case cannot occur. Hence, if $f \in \mathcal{U}_{2}$ is c-structurally stable then $f \in \mathcal{S} t_{2}$.

Assume now that $f \in \mathcal{V}_{2} \backslash \mathcal{U}_{2}$ is c-structurally stable. By its c-structural stability and Lemma 6.1, $f$ is conjugate to a c-structurally stable $g \in \mathcal{U}_{2}$. As we proved, this

$g$ belongs to $\mathcal{S} t_{2}$. By the definition of $\mathcal{S} t_{2}$, any map conjugate to a map from $\mathcal{S} t_{2}$ also belongs to $\mathcal{S} t_{2}$. Thus, $f \in \mathcal{S} t_{2}$.

Finally, by the definition of c-structural stability, the set of all c-structurally stable elements of $\mathcal{V}_{2}$ is open in $\mathcal{V}_{2}$. We will show that it is dense in $\mathcal{V}_{2}$. By Lemma 6.1 it is enough to show that it is dense in $\mathcal{U}_{2}$. However, this density follows immediately from Lemma 4.1, Theorem 4.3, and Theorem 5.3.

This completes the proof of Theorem 1.1.

\section{REFERENCES}

1. A. Blokh and M. Misiurewicz, Collet-Eckmann maps are unstable, Commun. Math. Phys. 191 (1998), 61-70.

2. J. Graczyk, D. Sands and G. Świątek, Metric attractors for smooth unimodal maps, Annals of Math. 159 (2004), 725-740.

3. P. Fatou, Sur les équations fonctionnelles, Bull. Soc. Math. France, 48 (1920), 33-94, 208-314.

4. M. V. Jakobson, Smooth mappings of the circle into itself (Russian), Mat. Sb. 85 (1971), 163-188.

5. O. S. Kozlovski, Axiom A maps are dense in the space of unimodal maps in the $C^{k}$ topology, Annals of Math. 157 (2003), 1-43.

6. O. S. Kozlovski, W. Shen and S. van Strien, Density of hyperbolicity in dimension one, Annals of Math. 166 (2007), 145-182.

7. G. Levin, S. van Strien, Bounds for maps of an interval with one critical point of inflection type II, Invent. Math. 141 (2000), 399-465.

8. M. Martens, Distortion results and invariant Cantor sets of unimodal maps, Ergodic Th. and Dyn. Syst. 14 (1994), 331-349.

9. W. de Melo and S. van Strien, "One-Dimensional Dynamics," Springer Verlag, Berlin 1993.

10. W. de Melo and S. van Strien, A structure theorem in one-dimensional dynamics, Annals of Math. 129 (1989), 519-546.

11. W. Shen, Decay of geometry for unimodal maps: An elementary proof, Annals of Math. 163 (2006), 383-404.

12. W. Shen, Decay of geometry - the $C^{2}$ case, preprint (2008).

13. M. Todd, One-dimensional dynamics: cross-ratios, negative Schwarzian and structural stability, PhD Thesis (2003), Univeristy of Warwick, Department of Mathematics.

Department of Mathematics, University of Alabama in Birmingham, University Station, Birmingham, AL 35294-2060

E-mail address: ablokh@math.uab.edu

Department of Mathematical Sciences, IUPUi, 402 N. Blackford Street, IndiANAPOLIS, IN 46202-3216

E-mail address: mmisiure@math.iupui.edu 